\title{
Low-cost spectrometers and learning applications for exposing kids to optics
}

Iman Khodadad, Navid Abedzadeh, Vasudevan Lakshminarayan, Simarjeet Saini

Iman Khodadad, Navid Abedzadeh, Vasudevan Lakshminarayan, Simarjeet S. Saini, "Low-cost spectrometers and learning applications for exposing kids to optics," Proc. SPIE 9793, Education and Training in Optics and Photonics: ETOP 2015, 97932W (8 October 2015); doi: 10.1117/12.2223241

Event: Education and Training in Optics and Photonics: ETOP 2015, 2015, Bordeaux, France 


\title{
LOW COST SPECTROMETERS AND LEARNING APPLICATIONS FOR EXPOSING KIDS TO OPTICS
}

Iman Khodadad $^{\text {a,c }}$, Navid Abedzadeh ${ }^{\text {a,c }}$, Vasudevan Lakshminarayan ${ }^{\text {b,c }}$ and Simarjeet S. Saini* a,c

${ }^{a}$ Department of Electrical and Computer Engineering, University of Waterloo, 200 University Ave W., Waterloo, ON, Canada; ${ }^{b}$ Optometry and Vision Science, University of Waterloo, 200 University

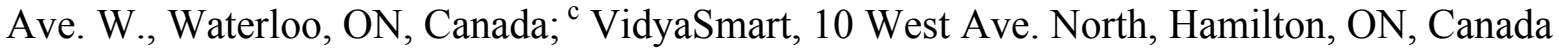

\begin{abstract}
We designed and built a low-cost imaging spectrometer using an in-house grating and a webcam and demonstrated its applications for active learning in science with experiments ranging from understanding light spectra from various sources to detecting adulteration in edible oils. The experiments were designed and run in an elementary school in Waterloo, Ontario with young students from grade 4 to grade 8 . The performance of the spectrometer is benchmarked to commercial spectrometers and showed excellent correlation for wavelengths between $450 \mathrm{~nm}$ to $650 \mathrm{~nm}$. The spectral range can be improved by removing infra-red filters integrated in webcams.
\end{abstract}

Keywords: Spectrometer, Light Sources, Fluorescence

\section{INTRODUCTION}

Organizing experiments with modern technologies and providing students the experience of installation, experimentation and execution prepares them to fulfill the requirements of contemporary sciences, production, investigation and design [1]. Kids begin to interact with light and colors at a very early age. This is often apparent from their fascination with rainbows. Thus, teaching about the properties of light through spectrometers is naturally exciting to kids. In Ontario curriculum composition of light from different colors is first taught in Grade 4 and according to the feedback from the teachers, the students have difficulty understanding how light breaks into different colors. Therefore, a low cost imaging spectrometer can be very useful for pedagogical approaches about light and optics. An imaging spectrometer for this purpose should be able to decompose light from different sources into various composite colors which the students can view and from which they can obtain spectral data to understand how light interacts with different media. Consequently, the students can explore engineering applications for light and optics. Such a spectrometer addresses students' interests from elementary to secondary schools. Hands-on learning through the use of spectrometers and carefully designed experiments will allow for:

1. Generation of interest in STEM fields

2. Appreciation for the functioning of technical devices in improving human well-being

3. Development of scientific temper and communication skills through presenting the findings

In recent years, there has been a growing interest in teaching spectroscopy at various educational levels [2-4]. A study done in Romania showed that physics experiments done with real instruments highly enhances the learning experience and increases the motivation in scientific exploration. The results with real experiments were significantly better than even virtual experiments as they "help to open the secrets of the world" [5]. In another study, it was found that the most effective learning happens not when the students are shown a demonstration but when they do the experiments themselves [6].

Educational spectrometers available today cost from a few hundred dollars to a few thousand dollars. The cheapest educational spectrometer, Vernier SpectroVis Plus, costs US $\$ 661$ and has a spectral resolution of $4 \mathrm{~nm}$ at $656 \mathrm{~nm}$ [7]. The spectrometer works well for measuring absorption or fluorescence spectra of liquids but cannot be used for much else. Public Labs has developed a very low cost Do-It-Yourself spectrometer kit which uses a DVD as the grating element and a housing made of cardboard which sells for US \$45. The spectrometers requires a trained person to assemble and works well for a DIY enthusiast. Further, measurements of samples require calibrations and careful mounting of the samples. There is a need for low cost spectrometers for the purpose of active learning, especially in the developing world. The low cost and the simplicity of the design makes this device readily accessible to publically funded

*sssaini@uwaterloo.ca; phone 15198884567 ext. 35018;

Education and Training in Optics and Photonics: ETOP 2015, edited by Eric Cormier, Laurent Sarger Proc. of SPIE Vol. 9793, 97932W · @ 2015 SPIE, IEEE, OSA, ICO · doi: 10.1117/12.2223241 


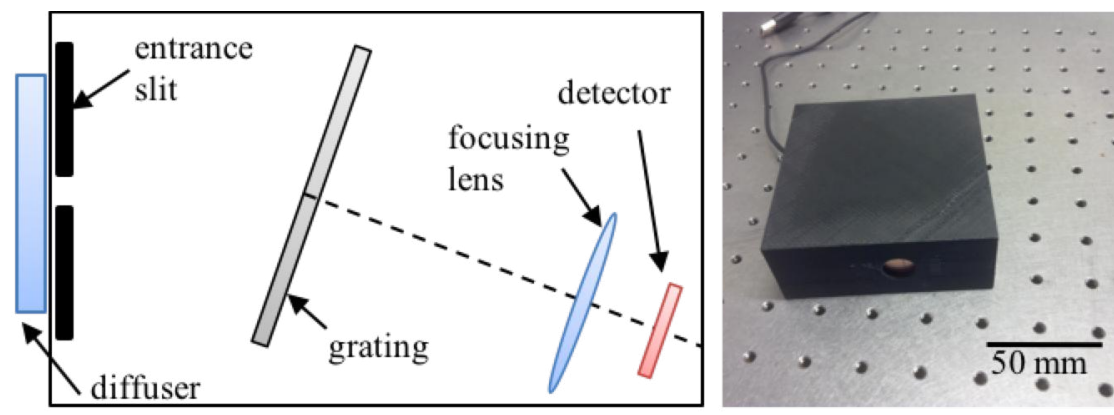

Figure 1. Schematics of the constructed spectrometer showing the simple design (left); spectrometer inside its housing (right)

schools particularly in the developing world. In this paper, we describe the design and experiments we conduct with our spectrometer. The spectrometer currently costs less than $\$ 50$ to build and the cost can be brought down to less than $\$ 20$ through mass production.

\section{CONSTRUCTION OF THE LOW COST SPECTROMETER}

The spectrometer uses our own home built volume phase holographic grating with 1800 lines/mm optimized for dispersion at $532 \mathrm{~nm}$. The grating works in transmission allowing for simple optical designs as shown in a schematic in Fig 1. The grating has a first order diffraction efficiency of $76 \%$ from $500 \mathrm{~nm}$ to $650 \mathrm{~nm}$. The grating absorbs light in the blue region which is currently being optimized. High efficiency grating allows us to use simple low cost optics; while achieving acceptable signal to noise ratio and spectral resolution. The slits are made with direct laser writing on a copper substrate with a width $100 \mu \mathrm{m}$. No collimating lens is used. The lens of the webcam is focused on the slit to image the slit with maximal resolution. A simple scotch tape based diffuser is used to diffuse incoming light when various light sources are measured and is easily removed for absorption and fluorescence experiments. As it can be seen from the image in Fig. 1, the spectrometer is $100 \mathrm{~mm} \times 100 \mathrm{~mm}$ in cross-section and $40 \mathrm{~mm}$ in height. The spectrometer housing is being redesigned to ergonomically fit in young kids hands. The spectrometer connects to android cell phones or tablets running an app or a laptop running a Labview software through a USB connection. Thus, the spectrometer allow for in and out of classroom portability and discovery. The webcam takes power from the cell phone and requires no battery of its own. Importance of field trips in college level education was discussed in [8] and the portability of our spectrometer allows for experimentation during field trips. A sample holder consisting of a cuvette holder and light sources including white light source and two laser pointers can be attached to the front of the spectrometer at the entrance slit for sample measurements. The light sources are also powered through the same USB connection. Thus, the spectrometer can be used for multiple applications and experiments including visualization of emitted or reflected spectra; and absorption and fluorescence measurements.

\section{HANDS-ON EXPERIMENTS FOR THE STUDENTS}

In this section, we describe a few of the experiments designed and performed with the constructed spectrometer that can promote various discussion topics in the classroom between the teachers and students. The topics expand from discussing various light sources and their emission spectra to detailed observations on light and matter interactions. Phenomena such as absorption, reflection and fluorescence can be studied and explained and their use in practical applications could be discussed. Such experiments fit nicely in the physics, chemistry and biology curriculum. There have been a total of approximately 50 experiments designed and conducted in the school. Here we describe a few pertinent ones.

\subsection{DIFFERENT SOURCES OF LIGHT}

Analyzing the emission spectra of various light sources such as the sun, incandescent light bulb, fluorescent tubes and flames leads to the understanding that total spectra are often made up of various spectral bands emitted from different 

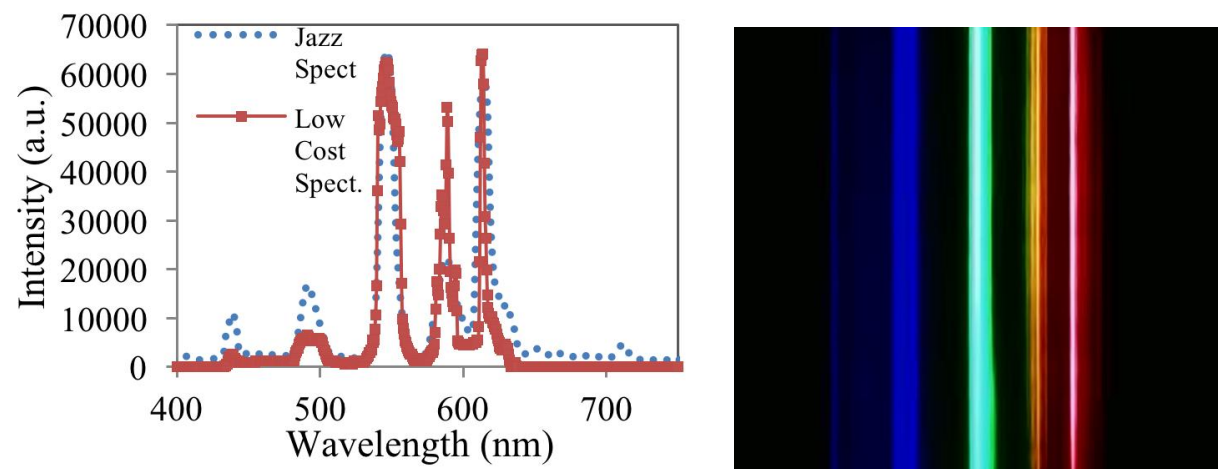

Figure 2. The spectrum of a flourescent tube source collected by the constructed low cost spectrometer (red) and the comparison with an Ocean Optics Jazz spectrometer (blue). The image taken by the webcam on the right shows the detailed colorful spectral lines.

molecules This would encourage the discussion of having many different molecule and atoms in the universe each of which would have their respective absorption and emission lines. Figure 2 shows the measured spectrum (left) along with the corresponding webcam image of the colors (right) from a fluorescent light tube. The spectral resolution achieved was $2.5 \mathrm{~nm}$, similar range to Vernier Spectra-plus. Using a collimating lens and a better slit enhanced the resolution to $0.3 \mathrm{~nm}$. As it can be seen from the colorful webcam image, the spectrum consists of discrete emission lines that are due to the presence of mercury in such sources. The spectra captured by the low cost spectrometer is calibrated and then compared to an Ocean Optics Jazz spectrometer. Although the resolution and dynamic range of the constructed spectrometer is not as good as that of Jazz spectrometer, major features of the spectrum are clearly resolved and comparable to the spectrum collected with Jazz. Hence at a fraction of the cost, the spectroscopic studies can be made widely accessible for educational purposes triggering scientific observation and discovery in and out of the classrooms. Figure 3 shows the spectral composition for various white light sources showing the students that the "white" color is comprised of many different spectral bands. This leads to a discussion of CIE color space. They can then measure the colors reflected off their clothes in different background lights and understand our perception of color.

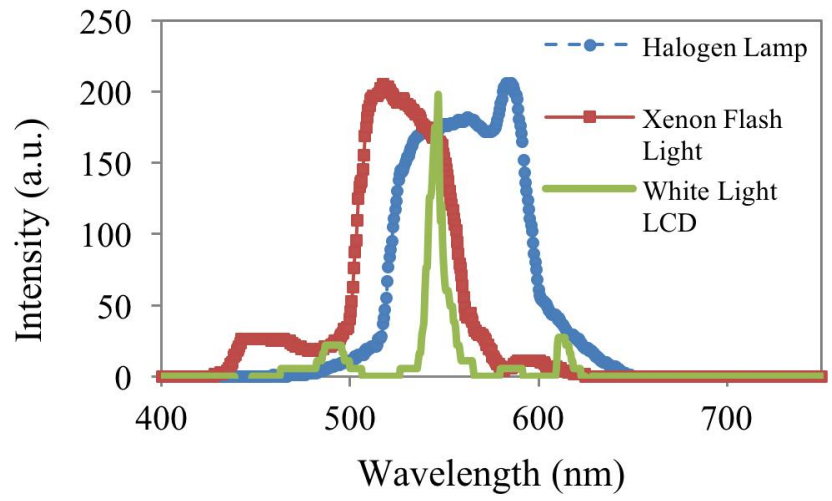

Figure 3. Spectra collected by the low cost spectrometer for various light sources.

\subsection{HOW LIQUID CRYSTAL DISPLAYS WORK}

All kid interact with liquid crystal displays (LCD) either through computers, TVs or tablets. One of the interesting experiments that can trigger many follow-up discussions is how modern LCD screens generate color. Students can use painting software to create areas with different colors on the screen and study the spectra of those colors to understand how each color can be constructed from primary colors of blue, green and red. Figure 4 shows the respective spectra of these various colors. In each of the spectral plots, the same spectrum was also measured using the Jazz spectrometer for comparison purposes. As it can be seen, the RGB filter response of the webcam as well as the dynamic range of its detector determine the quality of the reconstructed spectra. Hence, the low cost spectrometer has a limited ability in 

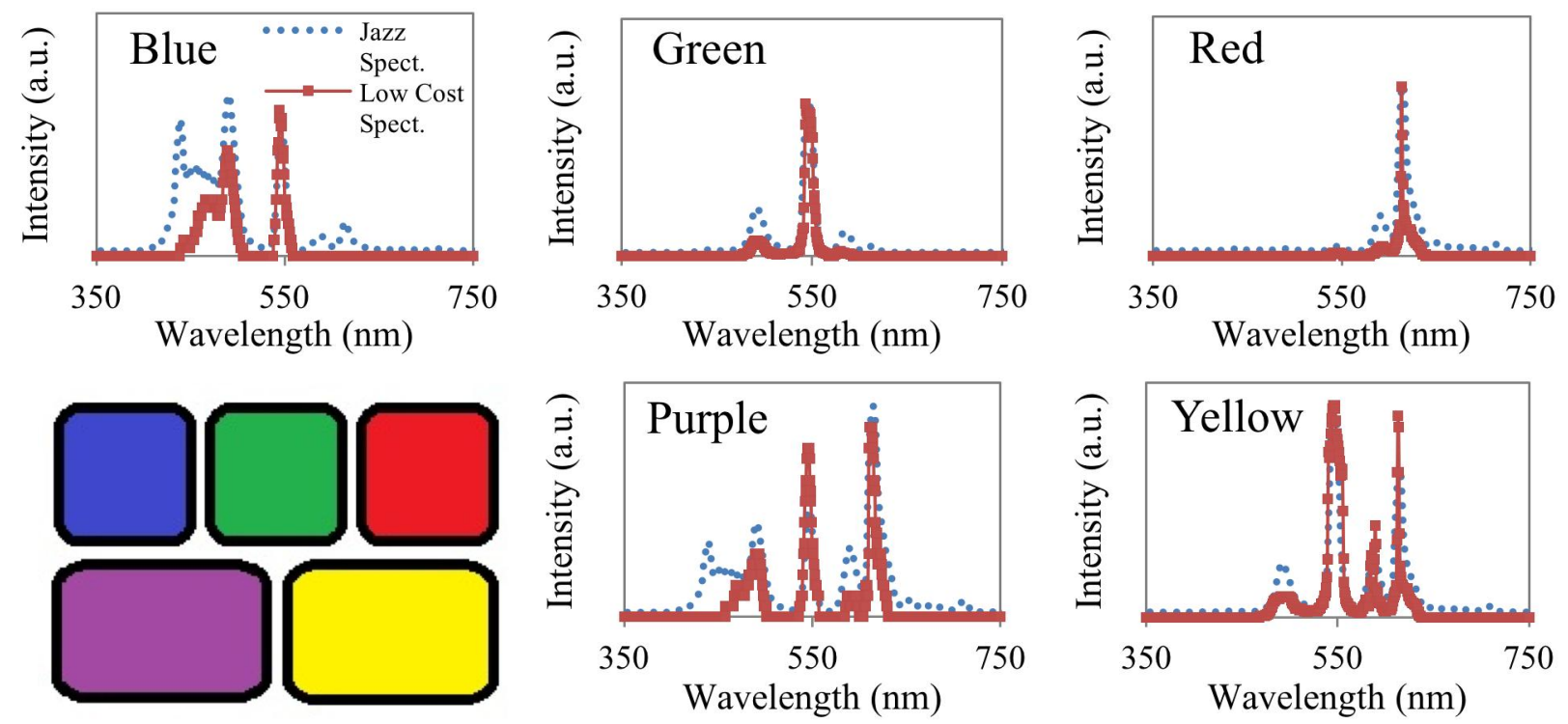

Figure 4. Spectra taken from an LCD screen on which various colors where shown at different parts of the screen. Their respective spectra are marked and shown in this figure. For comparison purposes, these colors' spectra were also measured by the Jazz spectrometer (blue dotted curves).

resolving and reconstructing all spectral features within the incoming light. For the combined colors such as yellow and purple, the overall spectrum consists of peaks in all three primary colors of blue, green and red indicating which filters within the LCD pixels are passing light through and by what amount.

\subsection{FLUORESCENCE IN EDIBLE OILS}

Edible oils are interesting subjects for educational studies. They are safe to use in the classroom and abundantly available. Edible oils such as olive oil and mustard oil show strong fluorescence in presence of $532 \mathrm{~nm}$ excitations. Hence, a green handheld laser diode can be used to show and study fluorescence. The phenomenon of fluorescence is seen in many applications such as phosphor fluorescence in fluorescent light tubes, biochemistry, medicine, gemology, mineralogy and forensics. Depending on the target class age, a variety of these applications can be explored and practiced starting with this simple experiment. As it can be seen from Fig. 5a, the olive oil in the cuvette fluoresces when subject to $532 \mathrm{~nm}$ excitation. The collimated green beam of the hand held laser pointer changes color to a yellowred hue as it passes through the oil. In a 90-degree angle setup, the spectrometer entrance slit can be directly positioned on the side of the cuvette. In this setup, the collected signal would have a signature from the laser as well as the fluorescence signal as shown in Fig. 5b. There are many educational observations that can be planned and carried with the following setup. For instance, students can take the effort for compare fluorescence power of various edible oils. While more viscous oils like olive oil and mustard oil show strong fluorescing power, oils such as sunflower oil show minimal fluorescing signal. Hence, the fluorescence signature of these oils can be explored and used to find adulteration in such oils. For instance, if the less desired rice-bran oil is added to olive oil, the respective fluorescence signature of olive oil would change. Figure $5 \mathrm{c}$ shows this change for the case of olive oil adulteration with $20 \%$ and $10 \%$ of ricebran oil. As it can be seen, the change in fluorescence signal can indicate the presence of adulterants in the test sample. The big spike in the captured spectra is from the excitation laser that has leaked into the spectrometer in the 90-degree setup. The broader signal above the $532 \mathrm{~nm}$ spike is due to the fluorescence of the oils and is sensitive to oils type and combination. 


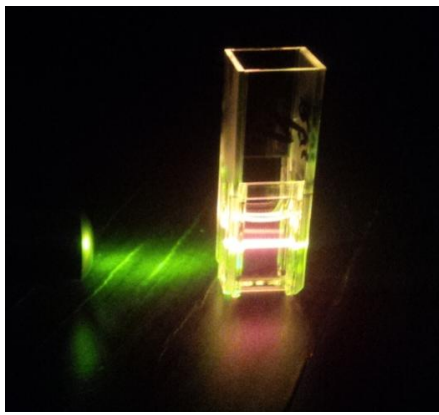

(a)

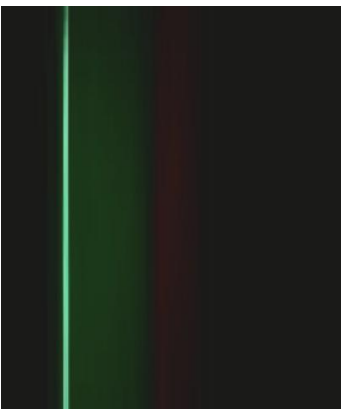

(b)

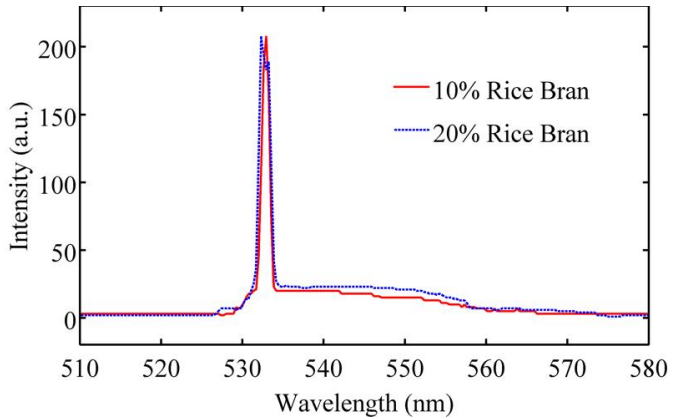

(c)

Figure 5. Fluorescence of edible oils. (a) Fluorescence of olive oil in presence of $532 \mathrm{~nm}$ excitation. (b) Captured spectra using the educational spectrometer. The green spike is the laser line followed by the longer wavelength fluorescence signal. (c) Comparison of the spectra collected using the low cost spectrometer from two adulterated samples of olive oil with $10 \%$ and $20 \%$ rice-bran oil.

\section{Conclusions and Future Plans}

We have developed a low cost spectrometer and designed multiple hands-on experiments for in and out of classroom active learning. The broad range of the designed experiments shows the versatility of this simply constructed spectrometer. The more visual experiments such as color composition of various light sources is aimed for students at lower grades where they would be mostly engaged through their visual sense. The more quantitative analysis of the spectra for fluorescence and absorption measurements can be used for various science experiments throughout higher education levels where students would learn to make comparative studies and logical deductions to arrive at scientific conclusions. Ergonomic designs are being considered for ease of use by young kids. Grating optimizations to improve the efficiency in the blue wavelengths are also being done. The spectrometers and the experiment plans would be available through VidyaSmart Inc. in Fall, 2015.

\section{REFERENCES}

[1] I. Bontaş, Treaty of Pedagogy, All Publishing, Bucharest, (2007).

[2] D. J. Butcher, Spectros. Lett., 43, 523 (2010).

[3] D. J. Butcher, Spectros. Lett., 40, 379 (2007).

[4] D. J. Butcher, R. G. Michel, Spectros. Lett., 37, VII (2004).

[5] I. M. Garabet, C. Miron, I. Neacsu, F. F. Popescu, "New educational perspectives on Spectroscopy in the Romanian High School", Romanian Reports in Physics, 64, 633-651 (2012).

[6] Cerghit I., Teaching methods, Didactical and Pedagogical Publishing House, Bucharest (1980).

[7] Vernier Software \& Technology, LLC http://www.vernier.com/products/sensors/spectrometers/visible-range/svis-pl

[8] K. Forest, S. Rayne, J. Chem. Educ., 86, 1290-1294 (2009). 